\title{
Measurement of the hadronic cross sections with the CMD-3 and SND detectors at the VEPP-2000 collider
}

A. A. Korol ${ }^{1,2, a}$, M. N. Achasov ${ }^{1,2}$, R. R. Akhmetshin ${ }^{1}$, A. N. Amirkhanov ${ }^{1,2}$, A. V. Anisenkov ${ }^{1,2}$, V. M. Aulchenko ${ }^{1,2}$, V. M. Aulchenko ${ }^{1,2}$, V. S. Banzarov ${ }^{1}$, A. Yu. Barnyakov ${ }^{1,2}$, N. S. Bashtovoy ${ }^{1}$, K. I. Beloborodov ${ }^{1,2}$, A. V. Berdyugin ${ }^{1,2}$, D. E. Berkaev1,2, D. E. Berkaev ${ }^{1,2}$, A. G. Bogdanchikov ${ }^{1}$, A. E. Bondar ${ }^{1,2}$, A. A. Botov ${ }^{1}$, A. V. Bragin ${ }^{1}$, T. V. Dimova ${ }^{1,2}$, V. P. Druzhinin ${ }^{1,2}$, S. I. Eidelman ${ }^{1,2}$, D. A. Epifanov ${ }^{1,2}$, L. B. Epshteyn ${ }^{1,2,3}$, A. L. Erofeev ${ }^{1,2}$, G. V. Fedotovich ${ }^{1,2}$, S. E. Gayazov ${ }^{1,2}$, V. B. Golubev ${ }^{1,2}$, A. A. Grebenuk ${ }^{1,2}$, S. S. Gribanov ${ }^{1,2}$, D. N. Grigoriev ${ }^{1,2,3}$, F. V. Ignatov ${ }^{1}$, V. L. Ivanov ${ }^{1,2}$, L. V. Kardapoltsev ${ }^{1,2}$, S. V. Karpov ${ }^{1}$, A. S. Kasaev1, V. F. Kazanin ${ }^{1,2}$, A. G. Kharlamov ${ }^{1,2}$, A. N. Kirpotin ${ }^{1}$, I. A. Koop ${ }^{1,2,3}$, I. A. Koop ${ }^{1}$, A. A. Korobov ${ }^{1,2}$, S. V. Koshuba1, D. P. Kovrizhin ${ }^{1,2}$, A. N. Kozyrev ${ }^{1,2}$, E. A. Kozyrev'1,2, P. P. Krokovny ${ }^{1,2}$, A. S. Kupich 1 , A. E. Kuzmenko1,2, A. S. Kuzmin ${ }^{1,2}$, I. B. Logashenko ${ }^{1,2}$, P. A. Lukin 1,2, A. P. Lysenko1, A. P. Lysenko1, K. A. Martin'1, N. A. Melnikova ${ }^{1,2}$, K. Yu. Mikhailovi,2, N. Yu. Muchnoi ${ }^{1,2}$, A. E. Obrazovsky ${ }^{1}$, V. S. Okhapkin ${ }^{1}$, E. V. Pakhtusova ${ }^{1}$, E. A. Perevedentsev ${ }^{1,2}$, E. A. Perevedentsev ${ }^{1,2}$, Yu. N. Pestov ${ }^{1}$, A. S. Popov ${ }^{1,2}$, G. P. Razuvaev ${ }^{1,2}$, Yu. A. Rogovsky ${ }^{1,2}$, Yu. A. Rogovsky ${ }^{1,2}$, A. A. Ruban ${ }^{1}$, N. M. Ryskulov ${ }^{1}$, A. E. Ryzhenenkov ${ }^{1,2}$, A. I. Senchenko ${ }^{1,2}$, S. I. Serednyakov ${ }^{1,2}$, P. Yu. Shatunov ${ }^{1,2}$, Yu. M. Shatunov ${ }^{1,2}$, Yu. M. Shatunov ${ }^{1}$, V. E. Shebalin ${ }^{1,2}$, D. N. Shemyakin ${ }^{1,2}$, D. A. Shtol ${ }^{1,2}$, B. A. Shwartz ${ }^{1,2}$, D. B. Shwartz ${ }^{1,2}$, A. L. Sibidanov ${ }^{4}$, Z. K. Silagadze ${ }^{1,2}$, A. N. Skrinsky ${ }^{1}$, E. P. Solodov ${ }^{1,2}$, I. K. Surin ${ }^{1,2}$, A. A. Talyshev ${ }^{1,2}$, Yu. A. Tikhonov ${ }^{1,2}$, Yu. V. Usovi,2, A. V. Vasiljev ${ }^{1,2}$, A. I. Vorobiov' ${ }^{1}$, Yu. V. Yudin ${ }^{1,2}$, and I M. Zemlyansky ${ }^{1,2}$

${ }^{1}$ Budker Institute of Nuclear Physics, SB RAS, Novosibirsk, 630090, Russia

${ }^{2}$ Novosibirsk State University, Novosibirsk, 630090, Russia

${ }^{3}$ Novosibirsk State Technical University, Novosibirsk, 630092, Russia

${ }^{4}$ University of Victoria, Victoria, British Columbia, Canada V8W 3P6

\begin{abstract}
Since December 2010 the CMD-3 and SND detectors detectors collect data at the VEPP-2000 electron-positron collider. In 2013-2015 the injection facility of the collider has undergone an upgrade of the injection system. The new BINP injection complex has been connected to the VEPP-2000 collider, so the restrictions connected to the lack of positrons and limited beam energy transfer do not apply any more. The collider luminosity in whole energy range is restricted now only by beam-beam effects. VEPP-2000 collider started to collect data with two detectors at 2016 year. The collected data sample since 2010 corresponds more than $100 \mathrm{pb}^{-1}$ of integrated luminosity per detector in the c.m. energy between 0.32 and $2 \mathrm{GeV}$. We will report here results of analysis of various hadronic cross sections from detectors both published and preliminary. These measurements are important by themselves and also because of the implications for anomaly of the magnetic moment of a muon (g-2) discrepancy.
\end{abstract}

a e-mail: a.a.korol@inp.nsk.su 


\section{Introduction}

Presented are the results of the analysis of data collected on the CMD-3 and the SND detectors installed in the interaction regions of the VEPP-2000 collider [1]. Collider can operate in c.m. energies $0.3-2.0 \mathrm{GeV}$. Luminosity delivered at $2 \mathrm{GeV}$ is $2 \times 10^{31} \mathrm{~cm}^{-2} \mathrm{~s}^{-1}$. During 2010-2013 experiments the luminosity however was limited by the shortage of positrons.

The Cryogenic Magnetic Detector (CMD-3) [2] (Fig.1) includes a tracking system consisting of a cylindrical drift chamber (DC) and a double-layer cylindrical multiwire proportional chamber (Zchamber) installed inside a thin superconducting solenoid with a $1.3 \mathrm{~T}$ field. The DC has $1.5-4.5 \%$ momentum accuracy for charged particles in the $100-1000 \mathrm{MeV} / \mathrm{c}$ momentum range, $20 \mathrm{mrad}$ for the polar $(\theta)$ angle and $3.5-8.0 \mathrm{mrad}$ for the azimuthal $(\varphi)$ angle. Ionization losses $\mathrm{dE} / \mathrm{dx}$ measured has the accuracy $11-14 \%$ minimum ionization particles. An electromagnetic calorimeter consists of the liquid xenon part (5.4X0 thickness) followed by the CsI crystals $(8.1 \mathrm{X0})$ outside of the solenoid in the barrel region and by the BGO crystals (14.4X0) in the endcap regions [3]. The scintillation counters for cosmic events veto are located outside the flux return yoke.

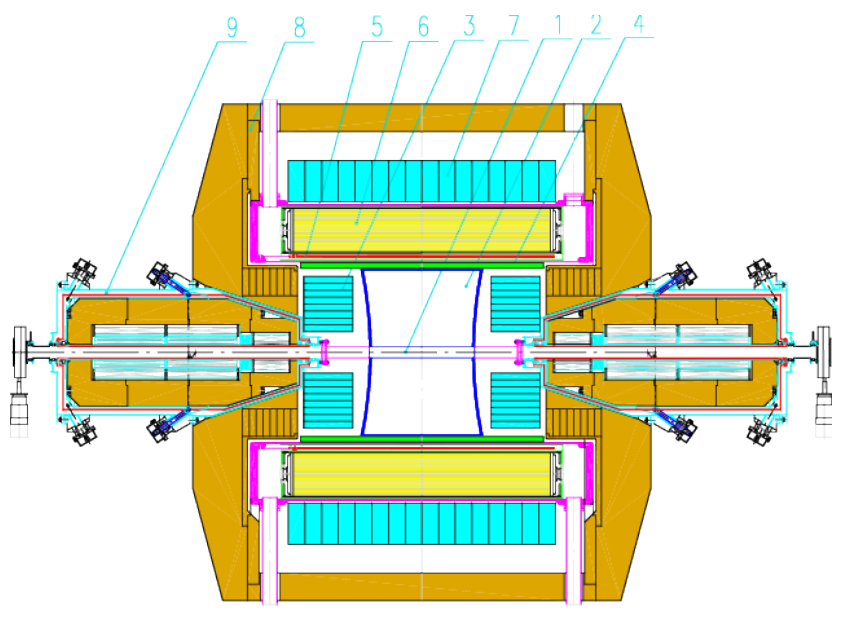

Figure 1. CMD-3 detector layout: 1 - vacuum chamber, 2 - drift chamber, 3 - electromagnetic calorimeter BGO, 4 - Z-chamber, 5 - CMD SC solenoid, 6 - electromagnetic calorimeter LXe, 7 - electromagnetic calorimeter CsI, 8 - yoke, 9 - VEPP-2000 solenoid

The Spherical Neutral Detector (SND) (Fig.2)[4, 5] includes a spherical electromagnetic calorimeter, a cylindrical tracking system, a threshold Cherenkov counters and a cosmic events veto detector. Electromagnetic calorimeter covers $0.95 \times 4 \pi$ of the solid angle, has $13.4 X 0$ thickness, its energy resolution $\frac{\delta E}{E} \sim \frac{4.2 \%}{\sqrt[4]{E(G e V)}}$ and angle resolution $\delta \varphi=\frac{0.82 \circ}{\sqrt{E(G e V)}} \oplus 0.63^{\circ}$. Tracking system covers $0.94 \times 4 \pi$ of the solid angle, its angle resolution $\delta \varphi=0.55^{\circ}, \delta \theta=1.2^{\circ}$ [6]. Cherenkov counters allows $K / \pi$ separation for $E<1 \mathrm{GeV}$.

Accurate VEPP-2000 beam energy measurement is provided by the system based on the Compton backscattered laser photons [7]. 


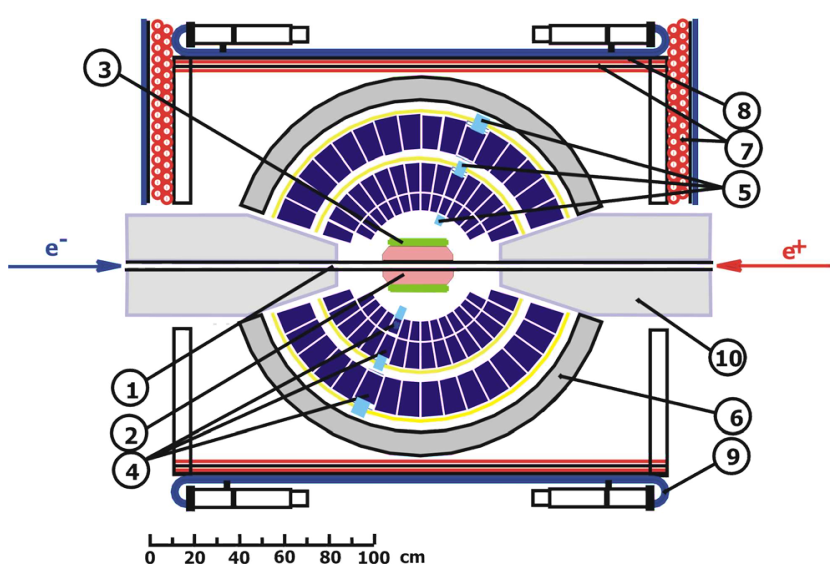

Figure 2. SND detector layout: 1 - beam pipe, 2 - tracking system, 3 - aerogel Cherenkov counter , 4 $\mathrm{NaI}(\mathrm{Tl})$ crystals, 5 - phototriodes, 6 - iron muon absorber, 7-9 - muon detector, 10 - focusing solenoids.

Physical program of detector experiments includes the following. (1) Study of dynamics of hadron production, i.e. separation between different intermediate states (like $\omega \eta, \varphi \eta, \rho a$ etc. in the reaction $e^{+} e^{-} \rightarrow \pi^{+} \pi^{-} \pi^{0} \eta$ ). This is needed for understanding hadronization mechanisms. (2) Hadron spectroscopy: study of light-vector-meson exitations, in particular, search for their radiative decays. (3) Search for rare and forbidden decays of the $\rho, \omega$, and $\varphi$ mesons. (4) Study of nucleon-antinucleon pair production, extraction of the proton and neutron electromagnetic formfactors. (5) Two-photon physics, in particular, measurement of the photon-meson transition form factors for $\pi^{0}, \eta, \eta^{\prime}$. (6) Search for production of C-even resonances: $e^{+} e^{-} \rightarrow \eta ; \eta^{\prime} ; f 1 ; f 2 ; a 2 \ldots$ (7) Using radiative return technique as alternative method for measurement of hadronic cross sections. (8) Measurement of exclusive hadronic cross sections below $2 \mathrm{GeV}$. The goal is to obtain the total cross section for $e^{+} e^{-} \rightarrow$ hadrons, which used for calculation HVP contribution to the muon $(g-2)$ and the running $\alpha_{Q E D}$. (9) Test of high-order QED: $2 \rightarrow 4,5$. (10) Other studies. Total statistics recorded for both detectors during 2010-2013 run is about $135 \mathrm{pb}^{-1}$, where $\sim 17 \mathrm{pb}^{-1}$ are recorder in the $\sim \omega$-region, $\sim 19 \mathrm{pb}^{-1}$ for energies below $1 \mathrm{GeV}$ ( except $\omega$-region), $\sim 17 p b^{-1}$ for $\varphi$-region, and $\sim 82 p b^{-1}$ above $\varphi$.

Recently (end of 2016) collider started operations after serious upgrade which promises to increase the luminosity with the help of the new injection complex [8]. Now the luminosity limited only with beam-beam effects.

\section{Hadronic processes}

\subsection{Process $e^{+} e^{-} \rightarrow \pi^{+} \pi^{-}$}

Now there is a certain discrepancy $(\sim 3.6 \sigma)$ between SM prediction and experimental result for anomalous magnetic dipole moment of a muon $\left(a_{\mu}=(g-2) / 2\right)$. The $e^{+} e^{-} \rightarrow \pi^{+} \pi^{-}$process is well known for its contribution $\left(\sim \frac{3}{4}\right)$ to the hadronic part $a_{\mu}$ [9]. Total cross hadronic cross-section of the whole VEPP-2000 energy region responsible for $92 \%$ of its value. The preliminary cross-section results was obtained for the process cross-section and related electromagnetic formfactor of $\pi$-meson from CMD-3 data analysis (Fig.3). Systematic error contributions now are radiative correction to 


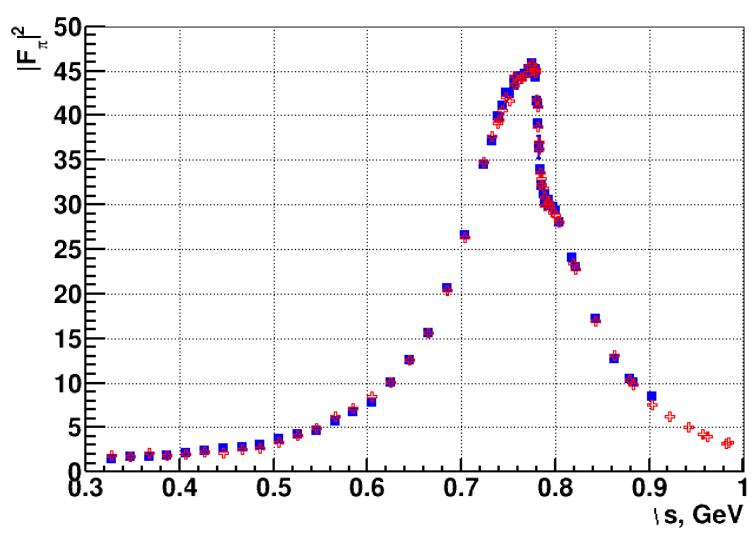

Figure 3. CMD-3: $\pi$-meson electromagnetic formfactor. Blue squares $-e / \mu / \pi$ separation by momentum, red crosses $-e / \mu / \pi$ separation by calorimeter energy deposition.

$0.2-0.4, e / \mu / \pi$ separation $0.1-0.5 \%$, pion decay and nuclear interaction $0.3-0.6 \%$. The goal is to reach full systematic error under $0.36 \%$. Statistical error varias from $\sim 0.3 \%$ near the $\rho$-meson mass to $3-7 \%$ at the edge of the region.

\subsection{Process $e^{+} e^{-} \rightarrow \pi^{0} \gamma$}

The $e^{+} e^{-} \rightarrow \pi^{0} \gamma$ has the third largest cross section in the c.m. energy region below $1 \mathrm{GeV}$, related with $\varphi, \omega$ and $\rho$ mesons radiative decays and the transition formfactor $\pi^{0} \gamma^{*} \gamma$. This cross section has been measured with SND detector [10] (Fig.4) using the full statistics collected at VEPP-2000 and previous VEPP-2M colliders. . The process $e^{+} e^{-} \rightarrow 2 \gamma$ is used for luminosity calculations. For analysis such selection criteria as charged track absence large total energy deposition, low momentum estimation in calorimeter and muon veto was used. Then after applying 4C kinematic fit and angles and recoil mass constraints number of signal events was determined from fit of $\pi^{0}$ shape in the recoil mass spectra. The $\rho, \omega, \varphi$ mesons radiative decays branching ratios are fitted from cross section data.

\subsection{Process $e^{+} e^{-} \rightarrow \pi^{+} \pi^{-} \pi^{0} \eta$}

Process $e^{+} e^{-} \rightarrow \pi^{+} \pi^{-} \pi^{0} \eta$ cross section is measured on both CMD-3 and SND detectors (Fig.5). These are the first measurements of this process. The intermediate states such as $\omega \eta, \varphi \eta$, structureless $\pi^{+} \pi^{-} \pi^{0} \eta$ and $a 0(980) \rho$ are also studied. The known $\omega \eta$ and $\varphi \eta$ contributions explain about 50-60\% of the cross section below $1.8 \mathrm{GeV}$. Above $1.8 \mathrm{GeV}$ the dominant reaction mechanism is $a 0(980) \rho$.

\subsection{Process $e^{+} e^{-} \rightarrow \omega \pi^{0} \rightarrow \pi^{+} \pi^{-} \pi^{0} \pi^{0}$}

Process $e^{+} e^{-} \rightarrow \omega \pi^{0} \rightarrow \pi^{+} \pi^{-} \pi^{0} \pi^{0}$ is studied with SND detector data. Preliminary cross section using the 2011-2012 data subset shown at Fig.6. Statistical error varies in 2-16\% depending of energy while systematic error $1-9 \%$ 

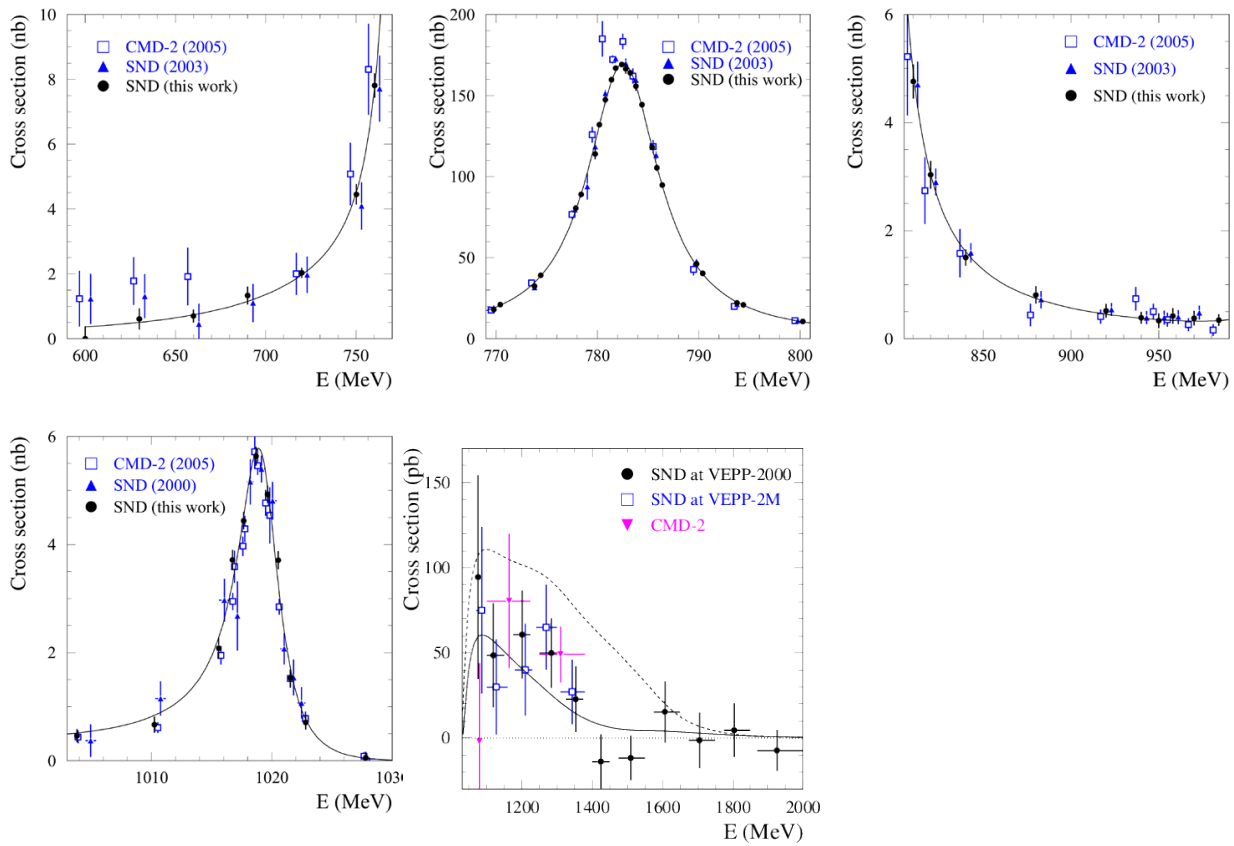

Figure 4. $e^{+} e^{-} \rightarrow \pi^{0} \gamma$ cross section for different energy ranges
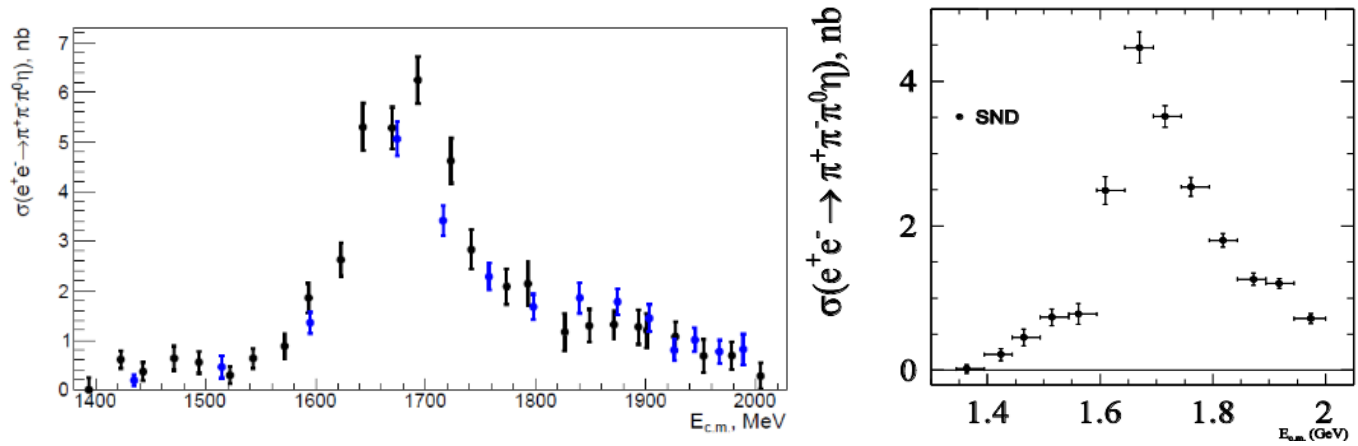

Figure 5. CMD-3 and SND process $e^{+} e^{-} \rightarrow \pi^{+} \pi^{-} \pi^{0} \eta$ cross section

\section{$2.5 e^{+} e^{-} \rightarrow 3 \pi^{+} 3 \pi^{-}$at the $N \bar{N}$ threshold}

One of the first result of CMD-3 2010-2012 data analysis was discovering of the noticeable decrease of the $e^{+} e^{-} \rightarrow 3 \pi^{+} 3 \pi^{-}$process cross section near the $N \bar{N}$ threshold (Fig.7). Preliminary data analysis for 2017 supported this observation. Depending of model this can be considered either as single transition or as two transitions near the $n \bar{n}$ and $p \bar{p}$ thresholds. More statistics is necessary to separate these cases. 


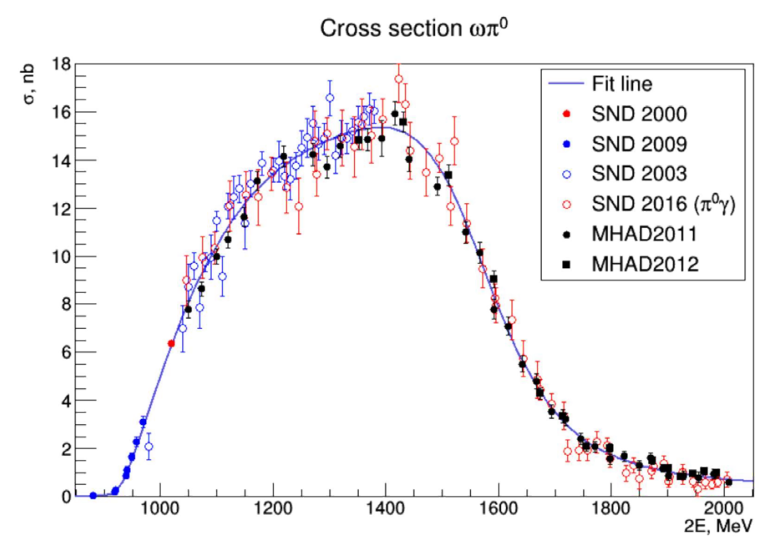

Figure 6. Process $e^{+} e^{-} \rightarrow \omega \pi \rightarrow \pi^{+} \pi^{-} \pi^{0} \pi^{0}$ cross section

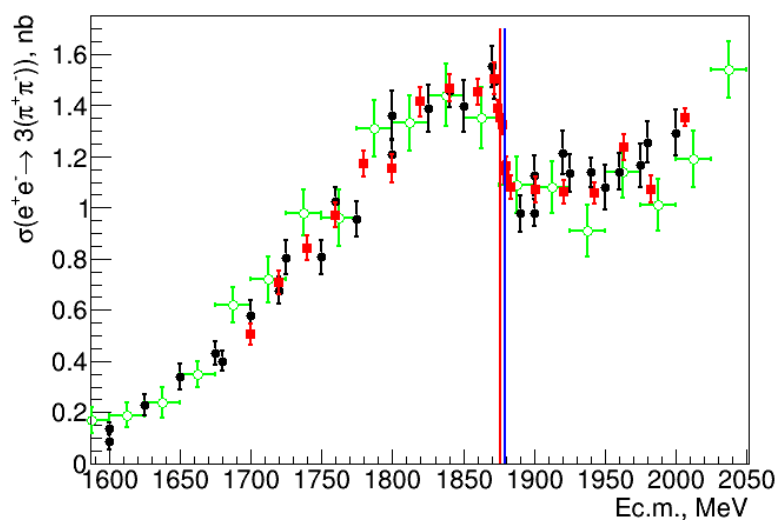

Figure 7. CMD- 3 cross section of $e^{+} e^{-} \rightarrow 3 \pi^{+} 3 \pi^{-}$process close to $N \bar{N}$ threshold. Black

\subsection{Processes $e^{+} e^{-} \rightarrow K^{+} K^{-}$and $e^{+} e^{-} \rightarrow K_{S} K_{L}$ near $\phi(1020)$}

Results of measurement of the $e^{+} e^{-} \rightarrow K^{+} K^{-}$and $e^{+} e^{-} \rightarrow K_{S} K_{L}$ [11] processes cross sections are shown at Fig.8. Used luminosity $6 \mathrm{pb}^{-1}$ Systematic error for $K_{S} K_{L}$ is $1.8 \%$. Systematic error for $K^{+} K^{-}$is $2.5 \%$ (preliminary).

\subsection{Process $e^{+} e^{-} \rightarrow K^{+} K^{-}$above $1.02 \mathrm{GeV}$}

Process $e^{+} e^{-} \rightarrow K^{+} K^{-}$cross section above $1.02 \mathrm{GeV}$ measured with SND data is shown at Fig.9. For final selection Cherenkov threshold counter is used. The complex shape of the cross section dependency is due to interference of many excited vector resonances in this energy region. 

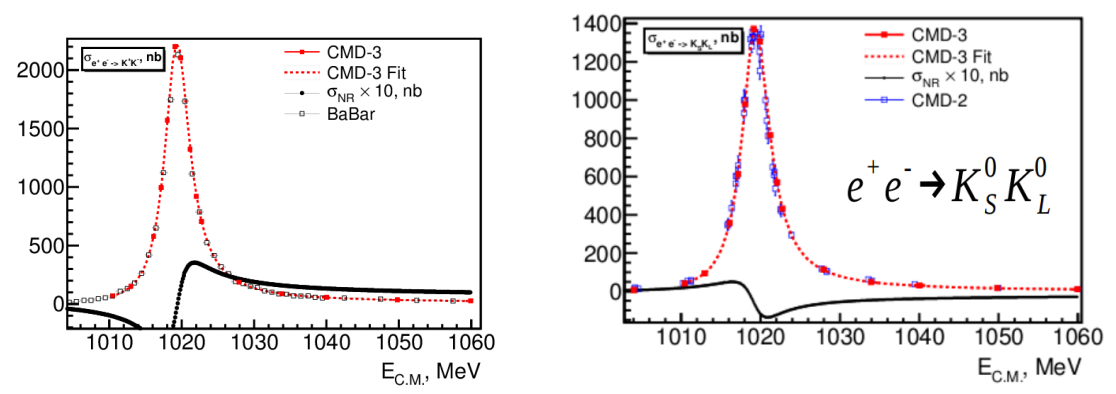

Figure 8. CMD-3 $e^{+} e^{-} \rightarrow K^{+} K^{-}$and $e^{+} e^{-} \rightarrow K_{S} K_{L}$ cross section

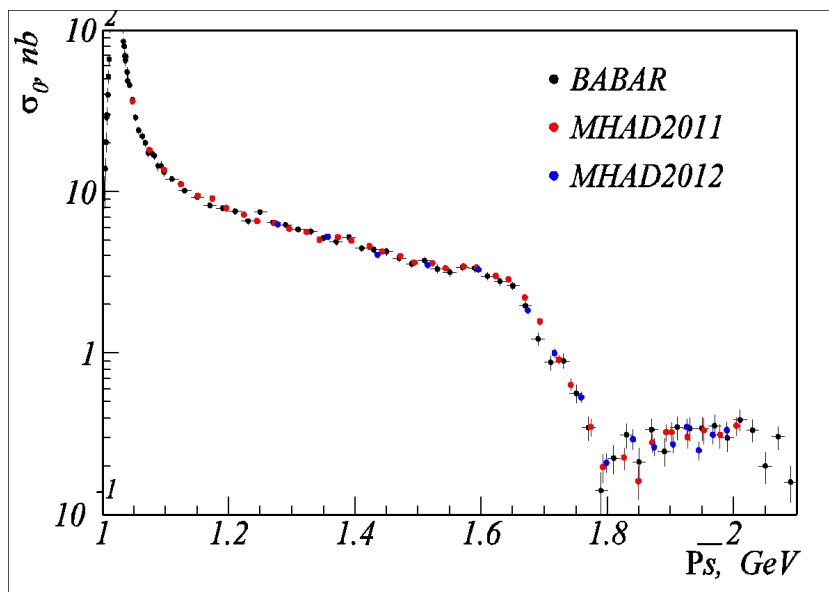

Figure 9. $\mathrm{SND} e^{+} e^{-} \rightarrow K^{+} K^{-}$above $\varphi(1020)$

\subsection{Process $e^{+} e^{-} \rightarrow K^{+} K^{-} \eta$}

Process $e^{+} e^{-} \rightarrow K^{+} K^{-} \eta$ cross section is measured for both CMD-3 and SND data (Fig.10). It is assumed that the dominant reaction mechanism is $\varphi(1680) \rightarrow \varphi(1020) \eta$. This hypothesis is in a good agreement with the data.

\subsection{Process $e^{+} e^{-} \rightarrow K^{+} K^{-} \pi^{0}$}

Process $e^{+} e^{-} \rightarrow K^{+} K^{-} \pi^{0}$ cross section is measured using CMD-3 data (Fig.11). Analysis is based on the integrated luminosity of $34 p b^{-1}$. Two intermediate states are clearly seen: $\varphi \pi^{0}$ and $K^{*}(892) K$ mechanism. The current systematic uncertainty is estimated as $10 \%$.

\section{Conclusions}

- During 2010-2013 both detectors accumulated $135 \mathrm{pb}^{-1}$ of integrated luminosity at the VEPP2000 electron-positron collider in the c.m. energy range $0.3-2.0 \mathrm{GeV}$. 

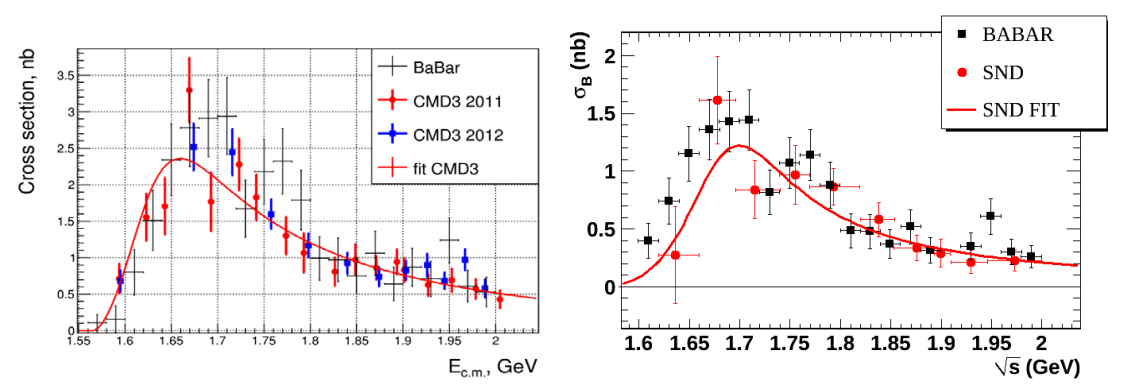

Figure 10. CMD-3 and SND $e^{+} e^{-} \rightarrow K^{+} K^{-} \eta$ cross sections

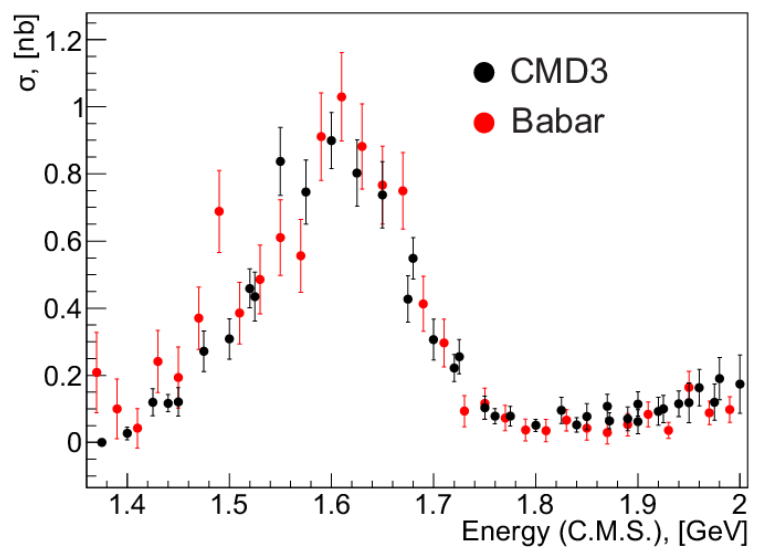

Figure 11. CMD-3 $e^{+} e^{-} \rightarrow K^{+} K^{-} \pi^{0}$ cross section

- Data analysis on hadron production is in progress. The obtained results have comparable or better accuracy than previous measurements.

- For some processes the cross sections have been measured for the first time.

- After VEPP-2000 upgrade the data taking runs are continued with a goal of $\sim 1 \mathrm{fb}^{-1}$ of integrated luminosity.

\section{Acknowledgements}

This work is supported in part by the RFBR grants 16-02-00327-a, 16-02-00014-a, 17-02-00327-a, 17-02-00847a, and 17-02-00897-a.

\section{References}

[1] B.I. Khazin (CMD-3, SND), Nucl. Instrum. Meth. A623, 353 (2010)

[2] B. Khazin, Nucl. Phys. Proc. Suppl. 181-182, 376 (2008) 
[3] V.E. Shebalin et al., Nucl. Instrum. Meth. A824, 710 (2016)

[4] M.N. Achasov et al., Nucl. Instrum. Meth. A449, 125 (2000), hep-ex/9909015

[5] G.N. Abramov et al., eConf C010430, T10 (2001), [,122(2001)], hep-ex/0105093

[6] V.M. Aulchenko et al., Nucl. Instrum. Meth. A598, 102 (2009)

[7] E.V. Abakumova et al., Nucl. Instrum. Meth. A744, 35 (2014), 1310.7764

[8] P.Yu. Shatunov et al., Phys. Part. Nucl. Lett. 13, 995 (2016)

[9] F. Jegerlehner, EPJ Web Conf. 118, 01016 (2016), 1511.04473

[10] M.N. Achasov et al. (SND), Phys. Rev. D93, 092001 (2016), 1601.08061

[11] E.A. Kozyrev et al. (CMD-3), Phys. Lett. B760, 314 (2016), 1604.02981 